\title{
Über die chemische Zusammensetzung des Axinits von Bourg d'Oisans in der Dauphine.
}

\author{
Von \\ P. Jannasch und James Locke.
}

Im Anschlufs an eine Reihe vor einiger Zeit publizierter Turmalinanalysen ${ }^{1}$ haben wir jetzt zwei genaue Analysen des Axinits von der Dauphiné ausgeführt, um damit einen weiteren Beitrag zur klareren Erkenntnis der Konstitution der Silikat-Borate zu liefern. Unser Hauptaugenmerk richteten wir auf eine möglichst sorgfältige und sichere Bestimmung des Wasser-, Borsäure-, Eisenoxydul- und Mangangehaltes nach praktisch erprobten Methoden, und in zweiter Linie suchten wir den zwar geringen, jedoch niemals fehlenden Alkaligehalt mit zweifelloser Bestimmtheit zu ermitteln.

Das Material zu den obigen Analysen von Bourg d'Oisans (Dauphiné) stammend, verdankten wir der Freundlichkeit des Hrn. Professor Liebisch in Göttingen, der selbiges auch mineralogisch begutachtete. Die Farbe der untersuchten schönen und völlig frischen Krystalle war mehr hell als dunkelamethystfarben und deren feines Pulver rein weifs. Das gröbliche Pulver schmilat in einem kleineren dünnwandigen Platintiegel sehr leicht, schon bei Anwendung einer gewöhnlichen hohen Gasflamme; die erhaltene Schmelze ist dunkelbraun und matt, in dünnen Schichten aber durchsichtig. Bei Anwendung der Gebläsespitzflamme tritt eine Aufblähung der Masse ein. Die Axinitschmelze ist vollständig löslich in konzentrierter Salzsäure.

Für die Analyse wurde das Mineral aufgeschlossen einmal mit einem Gemisch von Flufssäure und Schwefelsäure, sodann im geschmolzenen Zustande nur mit starker Salzsäure und drittens zum Zweck der einen Kieselsäurebestimmung und einer besonderen Manganwägung durch calcinierte Soda.

Bei der Silikataufschliefsung mit Flufssäure rühren wir das feine Pulver mit etwa $50 \mathrm{ccm}$ Wasser in einer mittelgrofsen Platinschale an, fügen 50-60 Tropfen $(5 \mathrm{ccm})$ konzentrierte Schwefelsäure hinzu, darauf die nötige Menge $(40-50 \mathrm{ccm})$ chemisch reine rauchende Flufssäure, dampfen auf dem Wasserbade ein, jagen den

1 Ber. deutsch. chem. Ges. 22, 216. 
Uberschuls an Schwefelsäure auf dem Lufbade ${ }^{1}$ fort und nehmen den Rückstand mit Wasser und reichlich viel konzentrierter Salzsäure auf $(15-20 \mathrm{ccm}$ Salzsäure, $150 \mathrm{ccm}$ Gesamtflüssigkeit und langeres Erwärmen auf dem Wasserbade). Diese Lösung wird nach vollständiger Oxydation des Eisens durch Zusatz von etwas konzentrierter Salpetersäure und Kochen im Becherglase in der Siedhitze mit einem mälsigen Überschuls ${ }^{2}$ von Ammoniak gefällt, der Niederschlag sofort filtriert und auf dem Filter 5-6 Mal gewaschen, wieder in überschüssiger Salzsäure gelöst und nochmals genau wie vorher gefällt, wodurch alles Mangan bis auf Spuren in die Lösung gelangt (cf. die speziellen Angaben hierüber weiter unten bei den analytischen Zahlen). Die Magnesia wird durch die gleichzeitige Gegenwart von viel Eisen unter den hier obwaltenden Mischungsverhältnissen nicht hartnäckig von den Sesquioxyden zurückgehalten, ${ }^{3}$ sondern befindet sich vollständig gelöst. In dem mit Essigsäure schwach angesäuerten und entsprechend eingeengten Filtrate von Eisen und Aluminium wird der Kalk durch alkalifreies Ammonoxalat ausgeschieden, nach dem Sammeln nochmals gelöst und von Neuem gefällt, worauf man die gesamte Flüssigkeit in einer grofsen Platinschale eintrocknet (zum Schlufs unter ununterbrochenem Rühren), danach zunächst auf einem offenen Luftbade etwa $1 / 2$ Stunde lang vorerhitzt und nun erst mit freier Flamme die Ammonsalze vorsichtig verjagt. Der Trockenrückstand wird in höchstens 20-25 ccm Wasser gelöst unter Zusatz von etwas verdünnter Essigsäure, in ein Becherglas gespült und jetzt die kochende Lösung zur Entfernung der Schwefelsäure mit einer gleichfalls kochenden Lösung von Bleiacetat gefällt und der Niederschlag wie iiblich weiter behandelt. Das alkoholische Filtrat rom Bleisulfat verdampft man in einem geräumigen Becherglase bis zur Verjagung des meisten Alkohols und fällt sodann aus der kochend heifsen Lösung den vorhandenen geringen Überschuls an Bleisalz durch einen raschen Schwefelwasserstoffistrom, bis sich das gebildete Bleisulfid vollkommen abgesetzt hat, worauf man filtriert, heifs auswäscht und die Flüssigkeit in einer Berliner Schale unter Zufügung von $5-i \mathrm{ccm}$ konzentrierter Salzsäure eintrocknet. Der Rest kommt dann gelöst in eine kleine Platinschale, oder Berliner Porzellan-

${ }^{1}$ Am saubersten für quantitative \%wecke sind aus dünnem Nickelblech geformte Becher (cf. Ber. deutsch. chem. Ges. 26, 1497).

a Juhrb. Mineral. (1888) 1, 196.

${ }^{3}$ Jabrb. Mineral. (1888) 1, 83. 
schale, um darin die Magnesia vermittelst Quecksilberoxyds abzuscheiden. Bei irgendwie reichlicheren Mengen von Magnesia (über $1 \%$ ) mufs die Quecksilberoxydbehandlung zweimal vorgenommen werden. Die Trennung von Kali und Natron kann nun in der bekannten Weise durch Platinchlorid erfolgen; man vergesse hier nicht, die beiden Alkalien vorerst noch einmal mit etwas freier Salzsäure zur Trockne zu bringen. Fürs Erste ist bei diesem Gange der Analyse für die Manganbestimmung zu bemerken, dals zunächst geringe Mengen dieses Metalles mit dem Kalk vereint fallen; man muls deshalb den im Platintiegel geglühten Ätzkalk in verdünnter Essigsäure (1:4) lösen, wobei die grölsere Menge des beigemengten Manganoxyds ungelöst zurückbleibt; aus der essigsauren, auf ca. $100 \mathrm{ccm}$ verdünnten Kalklösung werden am Ende die letzten in die Lösung übergegangenen Manganspuren mit Bromwasser bei Wasserbadwärme ausgeschieden. Das zweitens bei dem Quecksilberoxydverfahren restierende Gemisch von Manganoxyd und Mangnesia löst man in Salzsäure (Porzellanschale nehmen), trocknet ein, nimmt wieder in Wasser und wenig verdünnter Salzsäure auf, filtriert event. von Kieselsäure-Verunreinigung $a b$, neutralisiert das warme Filtrat mit Natriumkarbonat, bis ein bleibender Niederschlag entsteht, säuert mit verdünnter Essigsäure ${ }^{1}$ deutlich an und fällt endlich das Mangan mit Brom. ${ }^{2}$ Im Manganfiltrate wird das Magnesium mit Binatriumphosphat gefällt. Bei dem Magnesium ist die Hauptmenge des Mangans; nur ganz geringe Mengen desselben zeigen sich, wenn Eisen und Aluminium blofs zweimal mit Ammoniak gefällt wurden (cf. früher), auch noch bei den letzteren; trennt man nun diese durch die Natronschmelze im Silbertiegel, so werden die noch vorhandenen Manganspuren vollständig ${ }^{3}$ zu mangansaurem Natron oxydiert und

1 Wobei geringe Fällungen von unlöslichem basischen Aluminiam-EisenAcetat auftreten können, von denen man abfiltriert.

2 Anstatt das Mangan in der eben beschriebenen Weise an zwei Stellen (bei dem Kalk und der Magnesia) zu isolieren und zu bestimmen, würde es viel einfacher und rationeller sein, dasselbe direkt in dem ammoniakalischen Aluminium-Eisenfiltrat durch Wasserstoffwasseroxyd auszuscheiden. Zur Einführung des Verfahrens in die Silikatanalyse habe ich diese spezielle Fällung bei gleichzeitiger Gegenwart von Kalk- und Magnesiasalzen von Herrn V. WAsowICZ studieren lassen und hoffe eine baldige Mitteilung hierüber machen zu können. Die Ausfällung des Mangans mit Schwefelammonium ist nicht nur unbequem, sondern auch für den Verlauf des analytischen Ganges mit mancherlei Fehlerquellen behaftet und daher keinesfalls empfehlenswert.

${ }^{3}$ Das gewogene Gesamteisenoxyd wurde stets wieder gelöst und zur Kon- 
befinden sich so in der alkalischen Thonerdelösung. Erwärmt man die letztere in einer geräumigen Porzellanhenkelschale einige Zeit auf dem Wasserbade unter Zusatz von etwas Alkohol, so scheidet sich das Mangan wieder vollständig ab und kann gesammelt werden. Diese Abscheidung erfolgt auch für sich durch die blolse reduzierende Wirkung der in der natronalkalischen Flüssigkeit stets suspendiert vorhandenen Filtrierpapierflöckchen. ${ }^{1}$ Sämtliche bei dem Kalk, der Magnesia und den Sesquioxyden isolierten Manganmengen müssen noch einmal in gemeinschaftlicher essigsaurer Lösung durch Brom, oder durch Wasserstoffsuperoxyd in ammoniakalischer Lösung gefällt und vereint gewogen werden.

In einem dritten Falle wurde von uns der Mangangehalt des Axinits durch das Baryumkarbonatverfahren bestimmt. Wir beuutzten hierzu das salzsaure Filtrat der besonderen Kieselsäurebestimmung für die Analyse II. ${ }^{2}$ Diese Flüssigkeit dampften wir zunächst in einer Platinschale zur Entfernung der Salzsäure ein, lösten von neuem, neutralisierten die nur schwach saure, kalte Lösung beinahe mit Soda, fügten überschüssiges reines Baryumkarbonat hinzu und liefsen das Ganze bei gewöhnlicher Temperatur $\left(18-20^{\circ}\right)$ stehen unter öfterem Umschütteln. Man hüte sich vor dem Zusatz einer zu grofsen Menge des Baryumkarbonats, um die Niederschlagsmenge nicht ohne Not zu vermehren, da sich das entstehende Hydroxydgemisch nur äufserst schwierig auswaschen lärst (unter Umständen muls $35-40 \mathrm{mal}$ gewaschen werden). Selbstverständlich darf bei dieser Art der Trennung keine Schwefelsäure vorhanden sein, da sonst das Mangan mitfällt. Die erhaltenen Filtrate werden mit Essigsäure angesäuert, entsprechend konzentriert und am Ende mit Brom gefällt. Der Manganniederschlag wurde in Salzsäure wieder gelöst and in essigsaurer Lösung nochmals mit Brom ge-

trolle mit Kaliumpermanganat titriert. Wir haben aber niemals Differenzen gehabt, die auf einen gexingen Mangangehalt des Eisens hingedeutet hätten.

1 Unter Umständen kïnnen Spuren des durch die Natronschmelze fein zerteilten Eisenoxyds mit dureh das Filter gehen; man verhindert dieses aber sehr wirksam durch Auswaschung des Präparates mit sehr verdünnter Natronlange $(2 \%)$. Das thonordefreie Eisenoxyd wird wieder in Salzsäure gelöst, die Lösung von Verumreinigungen an Silberverbindungen abfiltriert und am Ende aus kochender Lösung mit einem grölseren Überschuls von Ammoniak gefällt, um vorhandenes Chlorsilber sicher in Lösung zu halten.

${ }^{2}$ Beim Verdampfen mit Flulssäure und sehwefelsäure hinterliefs diese Kieselsäure nur einen Rückstand von $0.0020 \mathrm{~g}$ 'Thonerde. Titansäure war nicht vorhanden; auch später bei Eisen und Thonerde fand sich keine Spur davon. 
fällt. Die zweimalige Fällung des Mangans mit Brom ist absolut notwendig, da der erste Niederschlag stark barythaltig ist und auch Kalk einschliefsen kann. Beim Auswaschen des mit Brom ausgeschiedenen Manganhyperoxydhydrats beobachtet man am Schluls häufig ein trübes Durchlaufen; diese Filtrattrübungen lassen sich durch Anwendung einer 10\% igen Ammonnitratlösung als Waschflüssigkeit leicht verhindern, oder man dampft die getrübten Filtrate etwas ein und erwärmt dann unter Bromzusatz so lange, bis sich die Teilchen vollkommen ablagern, worauf man dieselben für sich auf einem kleinen Extrafilterchen sammelt.

Zur Ausführung der Axinitanalyse durch einfaches Glühen des gröblichen Pulvers in einem Platintiegel und Lösung der erhaltenen Schmelze in Salzsäure verfährt man genau so, wie es früher der eine von uns für den Vesuvian und Kalk-Thonerdegranat des Näheren beschrieben. ${ }^{1}$ Die Lösung der Axinitschmelze in entsprechend konzentriert zu nehmender Salzsäure (1:1) erfolgt ziemlich rasch und leicht. Die in einer Platinschale befindliche Hauptmenge der Schmelze muls fleifsig mit dem Spatel gerührt werden. Die Lösung des Restes im bedeckt zu haltenden Platintiegel überlälst man bei Wasserbadhitze ruhig sich selbst; auch ist ein zeitweiliges Bedeckthalten der Platinschale anzuraten; ab und $\mathrm{zu}$ hat man das der Schmelze anhaftende Kieselsäurehydrat durch Drücken oder Stofsen zu entfernen. Die Hauptmasse der Kieselsäure findet sich gelöst, so dafs die Flüssigkeit beim Eindampfen sehr bald zu einer Gelatine erstarrt. I Lieses Verfahren der Axinitaufschliefsung ist offenbar das beste, sowohl im Interesse der erheblichen Vereinfachung der Analyse, wie zu Gunsten der grölseren Genauigkeit der Resultate. Zur Axinit-Sodaschmelze ist der Vollständigkeit wegen an dieser Stelle nur zu bemerken, dafs sich dieselbe rasch und fast ganz bei ihrer Zersetzung mit Salzsäure auflöst. Da hierbei ein deutlicher Chlorgeruch auftritt, so darf diese Operation nicht in einer Platinschale, sondern in einer Porzellanschale vorgenommen werden; später giebt man den Inhalt der letzteren wieder in die Platinschale zurück.

Die Bestimmung der Borsäure geschah nach dem von BodewIG verbesserten Marignacschen Verfahren. ${ }^{2}$ Da bei dieser Bestimmung die Beobachtung einer Menge von Vorsichtsmalsregeln eine nicht

1 Jahrb. Mineral. (1883) 2, 123.

${ }^{2}$ Grotns Zeitschr. Krystallogr. (1883) 8, 211, und Jahrb. Mineral. (1884) $9,14$. 
unbedeutende Rolle spielt, so liefern wir hier eine genauere Beschreibung der von uns angewandten Art ihrer Ausführung. Etwas iiber 1 s Substanz wurde mit der 6 fachen Menge an reinem calcinierten Kaliumkarbonat geschmolzen, zunächst über der gewöhnlichen Gasflamme (unvollkommene Schmelze) und dann kürzere Zeit vor dem Gebläse, wobei erst eine lebhafte Reaktion vor sich geht. Man lälst die Schmelze sich rasch abkühlen (Hineinhalten des noch ylühenden Tiegels in kaltes Wasser), da sie sich sonst schlecht vom Platin ablöst, behandelt sie mit heilsem Wasser (Platinschale), bis sie vollkommen zu Pulver zerfallen ist (Zerdrückung mit Glasstabpistill), und digeriert die Masse unter Zusatz von Fliefspapierschnitzeln (quantitativen Filtern entnommen) so lange, bis sich alle Mangansäure vollkommen zu unlöslichem Manganoxyd reduziert hat, die Flüssigkeit also entfärbt e'scheint, damit später das Magnesiumborat nicht etwa manganhaltig wird. Methylalkohol zur Reduktion des Kaliummanganats zu benutzen, fanden wir bedenklich gegenüber. einer event. Entstehung von flüchtigem Borsäure-Methyläther. Jetzt wird abfiltriert und mit kochendem Wasser anbaltend ausgewaschen, um sicher alle Borsäure in Lösung zu bekommen. Den unlöslichen Rückstand löst man probeweise in Salzsäure, um sich von der vollständig erfolgten Aufschlielsung der Substanz zu überzeugen und die salzsaure Lösung zu einer Probe auf Vorhandensein von Borsäure (mit Kurcumapapier) zu $\cdot$ unterziehen.

Zum alkalischen Bors:iurefiltrat fügt man jetzt $5-b \mathrm{~g}$ reinen Salmiak ( $4 / 5$ des angewandten Kaliumkarbonats) und 5-10 ccm konzentriertes Ammoniak und verdampft entsprechend, worauf man von der abgeschiedenen' Kieselsäure und Thonerde abfiltriert. Ist das Filtrat nicht absolut klar, so konzentriert man von neuem unter Ammoniakalischhalten und filtriert $\mathrm{zu}$ passender Zeit von einigen Flocken Niederschlag ab. $Z u$ dem letzteren Filtrat kommt nun das zur Überführung in Magnesiumborat erforderliche Magnesiumsal\% (die 14 fache Menge des mutmafslichen Borsäuregehaltes $=0.85$ - $1 \mathrm{~g} \mathrm{MgCl}_{2}+6 \mathrm{H}_{2} \mathrm{O}$ ), worauf konzentriertes Ammoniak zugesetzt und zur vollkommenen Trockne gebracht wird, am Schlufs unter stetem Rühren mit einem Platinspatel. Den Trockenrückstand erhitzt man anfänglich einige Zeit auf einem offenen Luftbade und zum Schluls erst mit freier Flamme zur Verjagung der Ammonsalze. $\mathrm{Zu}$ beachten ist bei den vorbeschriebenen Manipulationen vor allem, dafs bei dem Verdampfen die Flüssigkeit stets deutlich ammoniakalisch bleibt und niemals eine saure Beschaffenheit an- 
nimmt (Dissoziation des Ammoniumchlorids), weil damit sofort Borsäurererluste Hand in Hand gehen; ferner beachte man auch, dals nach dem Fortglühen der Ammonsalze bei möglichst klein zu wählender Flamme noch eine geraume Zeit $(1 / 4-1 / 2$ Stunde) mit voller Flamme geglüht wird, um dadurch die Borsäure recht vollständig in das unlösliche Magnesiumborat ïberzuführen. Den so geglühten Rückstand extrahiert man mit heifsem Wasser, filtriert $a b$ und wäscht den unlöslichen Teil auf einem kleinen Filter gut damit aus. Das Filtrat hiervon mufs noch einer Magnesiumbehandlung (mit $0.5-0.75 \mathrm{~g}$ krystallisiertem Chlorid) unterzogen werden unter erneutem Zusatz von 1.5--2 g Salmiak, Verdampfung der deutlich ammoniakalischen Flüssigkeit u. s. f. Die beiden das Magnesiumborat enthaltenden Filter werden im gewogenen Platintiegel verascht und die Präparate mit einem Glasstäbchen innig gemischt, mit Wasser durchtränkt, ron neuem getrocknet und geglïht bis zur Gewichtskonstanz. ${ }^{1}$ Von dem erhaltenen Magnesiumborat mufs eine Bestimmung der darin befindlichen Magnesia und der als Beimengung vorhandenen Kieselsäure, sowie eine Probe auf Chlorgehalt. gemacht werden. $\mathrm{Zu}$ der letzteren nimmt man nicht mehr als höchstens $0.05 \mathrm{~g}$, urn damit nach längerem Stehenlassen mit starker Salpetersäure, Verdünnung, Filtration etc. die Prüfung mit Silberlösung anzustellen. Handelt es sich nicht blofs um Spuren von Chlor (opalisierende Trübungen), so muls das ausgefallene Chlorsilber gewogen und in Rechnung gebracht werden. Die im Platintiegel verbliebene Hauptmenge an Magnesiumborat wird zurückgewogen und in einer Platinschale wiederholt mit Salzsäure eingedampft zur möglichsten Entfernung der Borsäure. Schliefslich trocknet man die krümlich gerührte Masse bei $108^{\circ}$, durchfeuchtet sie mit starker Salzsäure, verdünnt, erwärmt $1 / 4$ Stunde auf dem Wasserbade, filtriert und wägt den ungelöst gebliebenen Teil. Derselbe besteht aber nicht blofs, wie uns eine besondere Untersuchung auf Grund des metallgrauen Aussehens der Verbindung lehrte, aus Kieselsäure, sondern auch aus metallischem Platin. ${ }^{2}$

Die erhaltene salzsaure Magnesiumlösung fällt man unter den bekannten Vorsichtsmafsregeln mit Natriumphosphat. Wir raten, sich hierzu nur des gewöhnlichen Binatriumphosphates $\left(\mathrm{Na}_{2} \mathrm{HPO}_{4}\right.$ $\left.+12 \mathrm{H}_{2} \mathrm{O}\right)$ zu bedienen und nicht das neuerdings empfohlene soge-

2 Man achte hier auf einen Beschlag am Platintiegeldeckel (Kaliumchlorid!).

${ }^{2}$ Cf. hierüber eine spezielle Zahlenangabe bei Analyse II. 
nannte Phosphorsalz $\left(\mathrm{Na}_{\mathrm{NH}} \cdot \mathrm{HPO}_{4}+4 \mathrm{H}_{2} \mathrm{O}\right)$ zu nehmen. Wir haben gerade mit letzterem Doppelsalze schlechte Erfahrungen gemacht, denn diese Fällung gab meist viel zu hohe Zahlen und erst die Wiederlösung des Niederschlages in Salzsäure und dessen nochmalige Ausseheidung mit Ammoniak unter Zusatz von nur ein paar Tropfen Phosphorsalzlösung lieferte konstante Werte. Es scheint also das Ammonium-Natrium-Phosphat in etwas grölserem Überschufs vorhanden unter Bildung komplizierterer Doppelverbindungen teilweise von dem Magnesiumniederschlage mitgerissen zu werden. ${ }^{1}$ Ein solches Gemisch zeigt dann beim Glühen im Platintiegel die Eigenschaften des Schmelzens. Die im obigen ausführlich beschriebene Methode der Borsäurebestimmung ist zwar umständlich, liefert aber völlig zuverlässige, konstante Resultate und lohnt somit wenigstens den damit verbundenen grofsen Zeitaufwand.

Mit der vor einiger Zeit von GoocH vorgeschlagenen Bestimmung der Borsäure durch Isolieren derselben als Borsäure-Methyläther ${ }^{2}$ haben wir noch keine besonderen Versuche angestellt, gedenken aber diese Methode bei weiteren Axinitanalysen ebenfalls genauer für unseren Zweck zu studieren.

Die Ermittelung des Eisenoxydulgehaltes wurde einmal nach der Methode von Mrtschentich durch Aufschliefsen des sehr feinen Pulvers im geschlossenen Rohr mit konzentrierter Schwefelsäure (4 Teile : 1 Teil $\mathrm{H}_{2} \mathrm{O}$ ) und das andere Mal durch das Verfahren von Pebau-Doezter (Aufschlielsung mit Schwefelsäure-Flul'ssäure in einer Kohlensäureatmosphäre) ${ }^{3}$ bestimmt. Die letztere Methode giebt sehr genaue Resultate und ist, besonders in der Gesteinanalyse, einer weit allgemeineren Anwendung fähig als die von MitsCHERLICH gegebene Vorschrift, da nach anderweitigen Erfahrungen die konzentrierte Schwefelsäure unter Druck nicht immer vollkommen aufschliefsend auf gewisse Silikate einzuwirken scheint. ${ }^{4}$ Bei der Aufschliefsung der Silikate nach PeiBaL-Doelter sehe man besonders darauf, das Material recht fein gepulvert anzuwenden, den dazu er-

1 Es ist nicht ausgeschlossen, dals zu diesem Verhalten etwa noch vorhandene Borsäure mit beiträgt, und wollen wir bei späteren Analysen darauf achten.

2 Chem. News 55, 7.

3 Tschermaks mineralog. und petrogr. Mitteil. Jahrg. 1877, 281 und daselbst $1880,100$.

4 Cf. J. Fraycib Wildams, Jahrb. Mineral. 1877, V. Beilage-Band, Seite 405 . 
forderlichen Platintiegel nicht zu klein zu nehmen und später zur Verjagung der überschüssigen Flufssäure nicht länger, als unbedingt nötig, zu erhitzen, um die Bildung eines in verdünnter Schwefelsäure ganz unlöslichen, in schönen Blättern sich ausscheidenden Doppelsulfates von Aluminium und Eisenoxydul zu verhindern. Es mufs bei dem Eintragen des erkalteten Tiegelinhaltes in eine grölsere Menge Wasser fast vollständige Lösung eintreten als Zeichen eines normalen Verlaufes des chemischen Vorganges.

Die Ermittelung des Wassergehaltes geschah nach der von dem einen von uns herrührenden direkten Bestimmungsmethode, nämlich durch Schmelzen des feinen Pulvers mit Bleichromat in einem kurzen Verbrennungsrohr und Auffangung des freiwerdenden Wassers in einem gewogenen Chlorcalciumrohr. ${ }^{1}$ Bei gewissenhafter und geschickter Beobachtung aller in der citierten Abhandlung aufgeführten Einzelheiten liefert dieses Verfahren vollständig genaue Resultate.

\section{Analytische Resultate.}

I. Analyse. Lösung der Axinitschmelze in Salzsäure. $-1.1302 \mathrm{~g}$ Substanz gaben $=0.4846 \mathrm{~g} \mathrm{SiO}_{2}(42.88 \%) ; 0.2064 \mathrm{Al}_{2} \mathrm{O}_{3}(18.26 \%) ; 0.0966$ Gesarnteisenoxyd; $0.0135 \mathrm{Mn}_{3} \mathrm{O}_{4}{ }^{2}=0.0126 \mathrm{MnO}(1.11 \%) ; 0.2251 \mathrm{CaO}(19.92 \%) ; 0.0688$ $\mathrm{Mg}_{2} \mathrm{P}_{2} \mathrm{O}_{7}=0.0248 \mathrm{MgO} ; 0.0060 \mathrm{~K}_{2} \mathrm{PtCl}_{6}=0.0014 \mathrm{KCl}=0.00088 \mathrm{~K}_{2} \mathrm{O}(0.08 \%)$ und $0.0084 \mathrm{Na}_{2} \mathrm{SO}_{4}=0.0037 \mathrm{Na}_{2} \mathrm{O}(0.32 \%)$.

Eisenoxydulbestimmung nach Pẹal-Doenter. - $0.6524 \mathrm{~g}$ brauchten in Sulfatlösung $=6.5 \mathrm{ccm} 1 / 10$ Kaliumpermanganatlösung bis zur bleibenden Rotfärbung $=0.0364 \mathrm{~g} \mathrm{Fe}=0.0468 \mathrm{FeO}=0.0520 \mathrm{Fe}_{2} \mathrm{O}_{3}=7.17 \% \mathrm{FeO}$. Die letztere Menge $\mathrm{Fe}_{2} \mathrm{O}_{3}$ auf $1.1302 \mathrm{~g}$ Substanzmenge berechnet, entspricht $=0.0901 \mathrm{~g}$ $\mathrm{Fe}_{2} \mathrm{O}_{3}$, folglich $0.0966 \mathrm{~g}$ Gesamteisenoxyd $-0.0901=0.0065 \mathrm{~g} \mathrm{Fe}_{2} \mathrm{O}_{3}(0.58 \%)$ im Axinit.

Wasserbestimmung nach der Bleichromatmethode. $-0.9090 \mathrm{~g}$ lieferten $=0.0198 \mathrm{~g} \mathrm{H}_{2} \mathrm{O}=2.18 \%$.

Glühverlust. $-0.6964 \mathrm{~g}$ verloren bei kleiner Flamme $=0.0062 \mathrm{~g}(0.89 \%)$, bei voller Flamme $=0.0064 \mathrm{~g}=0.92 \%$ und vor dem Gebläse $=0.0094 \mathrm{~g}$ $=1.35 \%$.

Borsäurebestimmung. - $1.1182 \mathrm{~g}$ gaben 0.5116 Magnesiumborat inklusive $\mathrm{SiO}_{2}$ und $\mathrm{Pt}$; davon wurden $0.0426 \mathrm{~g}$ auf $\mathrm{Cl}$ geprüft (dieselben enthielten nur unwägbare Spuren); der Rest von 0.4609 Magnesiumborat gab bei der Analyse $0.0469 \mathrm{~g} \mathrm{SiO}_{2}+\mathrm{Pt}=0.0512 \mathrm{~g}$ für die Gesamtmenge und ferner $1.0013 \mathrm{~g}$ $\mathrm{Mg}_{2} \mathrm{P}_{2} \mathrm{O}_{7}=0.3608 \mathrm{MgO}=0.3936 \mathrm{~g}$ für das Ganze; $0.5116-0.4448(0.3936+0.0512)$ $=0.0668 \mathrm{~g}$ Borsäuredifferenz $=5.97 \%$.

1 Ber. deutsch. chem. Ges. 22, 221 und Mitteilungen der Grossh. Badisehen Geologischen Landesanstalt 2, 252.

${ }^{2}$ Bei dem Ätzkalk befanden sich 0.0042 , bei der Magnesia $=0.0083$ und bei Eisen und Aluminium $=0.0010 \mathrm{~g} \mathrm{Mn}_{8} \mathrm{O}_{4}$. 
II. Analyse. - A. Besondere Kieselsäurebestimmung mit Manganbestimmung. $1.1172 \mathrm{~g}$ gaben mit Soda aufgeschmolzen $=0.5028 \mathrm{~g} \mathrm{SiO}$ $=42.89 \%$ und $0.0139 \mathrm{~g} \mathrm{Mn}_{3} \mathrm{O}_{4}=0.0129 \mathrm{MnO}=1.10 \%$ (III. Manganbestimmung).

B. A f fschliefsung des Minerals durch Flufssäure und Schwefelsäure. $-1.1312 \mathrm{~g}$ gaben $=0.2062 \mathrm{~g} \mathrm{Al} \mathrm{A}_{2} \mathrm{O}_{3}(18.23 \%) ; 0.0960 \mathrm{Fe}_{2} \mathrm{O}_{8} ; 0.2245$ $\mathrm{CaO}(19.85 \%) ; 0.0123 \mathrm{Mn}_{3} \mathrm{O}_{4}=0.0114 \mathrm{MnO}(1.01 \%)$ [bei dem $\mathrm{CaO}$ waren $0.0028 \mathrm{~g}$ und bei der $\mathrm{MgO}=0.0095 \mathrm{~g} \mathrm{Mn}_{3} \mathrm{O}_{4}$ verblieben]; $0.0711 \mathrm{Mg}_{2} \mathrm{P}_{2} \mathrm{O}_{7}=$ $0.0256 \mathrm{~g} \mathrm{MgO}(2.26 \%) ; 0.0086 \mathrm{~g} \mathrm{~K}_{2} \mathrm{PtCl}_{6}=0.0026 \mathrm{KCl}=0.0016 \mathrm{~K}_{2} \mathrm{O}(0.14 \%)$ und $0.0100 \mathrm{Na}_{2} \mathrm{SO}_{4}=0.0044 \mathrm{Na}_{2} \mathrm{O}(0.39 \%)$.

Eisenoxydulbestimmung nach Mrschersich im geschlossenen Rohr. $-0.6950 \mathrm{~g}$ erforderten bei der Titrierung mit ${ }^{1} / 10 \mathrm{KMnO}_{4}=6.81 \mathrm{ccm}$ $=0.03810 \mathrm{Fe}=0.04899 \mathrm{FeO}=0.05443 \mathrm{Fe}_{2} \mathrm{O}_{3}=7.05 \% \mathrm{FeO}$. Die letztere $\mathrm{Fe}_{2} \mathrm{O}_{3}$-Menge auf $1.1312 \mathrm{~g}$ Substanz berechnet, ergiebt $=0.0886 \mathrm{~g}$, folglich 0.0960 Gesamteisen $-0.0886=0.0074 \mathrm{~g} \mathrm{Fe}_{2} \mathrm{O}_{3}=0.65 \%$ Eisenoxydgehalt für den Axinit.

Wasserbestimmung nach der Bleichromatmethode. - $1.1162 \mathrm{~g}$ gaben $=0.0234 \mathrm{~g} \mathrm{H}_{2} \mathrm{O}=2.10 \%$.

Borsäurebestimmung. $-1.2150 \mathrm{~g}$ gaben $=0.4554 \mathrm{~g}$ Magnesiumborat (inkl. $\mathrm{SiO}_{2}+\mathrm{Pt}$ ); davon wurden $0.0358 \mathrm{~g}$ zur Probe auf Chlor entnommen (nur eine Spur da); der Rest von $0.4196 \mathrm{~g}$ lieferte $=0.0375 \mathrm{~g} \mathrm{SiO}_{2}+\mathrm{Pt}^{1}=0.0407 \mathrm{~g}$ in Summa und ferner $0.8717 \mathrm{~g} \mathrm{Mg}_{2} \mathrm{P}_{2} \mathrm{O}_{7}=0.3141 \mathrm{MgO}=0.3409 \mathrm{~g}$ im ganzen; somit gaben die obigen $0.4554 \mathrm{~g}$ Magnesiumborat $-0.3816 \mathrm{~g} \mathrm{MgO}+\mathrm{SiO}_{2}+\mathrm{Pt}$ $=0.0738 \mathrm{~g}$ Borsäuredifferenz $=6.07 \%$.

Bestimmung des spezifischen Gewichtes. - 2.6856 verloren im Pyknometer bei $22^{\circ}$ C. $=0.8218 \mathrm{~g}=3.268$ spez. Gew.

Zusammenstellung und Ausrechnung.

\begin{tabular}{l|r|r|r|r}
\hline & \multicolumn{1}{|c|}{ I } & II & Mittel & $\begin{array}{r}\text { Auf } 100 \\
\text { berechnet }\end{array}$ \\
\hline \hline $\mathrm{SiO}_{2}$ & 42.88 & 42.89 & 42.88 & 42.60 \\
$\mathrm{~B}_{2} \mathrm{O}_{3}$ & 5.97 & 6.07 & 6.02 & 5.98 \\
$\mathrm{Al}_{2} \mathrm{O}_{3}$ & 18.26 & 18.23 & 18.24 & 18.12 \\
$\mathrm{Fe}_{2} \mathrm{O}_{3}$ & 0.58 & 0.65 & 0.62 & 0.62 \\
$\mathrm{FeO}$ & 7.17 & 7.05 & 7.10 & 7.06 \\
$\mathrm{MnO}$ & 1.11 & 1.01 & 1.06 & 1.05 \\
$\mathrm{CaO}$ & 19.92 & 19.85 & 19.89 & 19.76 \\
$\mathrm{MgO}$ & 2.19 & 2.26 & 2.23 & 2.22 \\
$\mathrm{~K}_{2} \mathrm{O}$ & 0.08 & 0.14 & 0.11 & 0.10 \\
$\mathrm{Na}_{2} \mathrm{O}$ & 0.32 & 0.39 & 0.36 & 0.36 \\
$\mathrm{H}_{2} \mathrm{O}$ & 2.18 & 2.10 & 2.14 & 2.13 \\
\hline & 100.66 & 100.64 & 100.65 & 100.00 \\
\hline
\end{tabular}

${ }^{1}$ Dieselben hinterliefsen nach Behandlung mit Flufssäure und Schwefelsäure $=0.0126 \mathrm{~g}$ metallisches Platin, das durch Lösen in Königswasser und Fällung mit Kaliumehlorid als solches charakterisiert werden konnte. 


\begin{tabular}{|c|c|c|c|}
\hline & $\%$ & & \\
\hline $\mathrm{Si}$ & 19.8970 & 0.7106 & $=0.7106 \mathrm{Si}$ \\
\hline B & 1.8705 & 0.1716 & \\
\hline Al & 9.6110 & 0.3554 & $=0.5348 \mathrm{R}^{\mathrm{II}}$ \\
\hline $\mathrm{Fe}^{\mathrm{lII}}$ & 0.4340 & 0.0078 & \\
\hline $\mathrm{Fe}^{I I}$ & 5.4912 & 0.0983 & \\
\hline $\mathrm{Mn}$ & 0.8134 & 0.0148 & $=0.5244 \mathrm{R}^{\mathrm{II}}$ \\
\hline $\mathrm{Ca}$ & 14.1146 & 0.3537 & $=0.0244 \mathrm{~K}^{\mathrm{I}}$ \\
\hline $\mathrm{Mg}$ & 1.3320 & 0.0556 & \\
\hline $\mathbf{K}$ & 0.0839 & 0.0021 & \\
\hline $\mathrm{Na}$ & 0.2672 & 0.0116 & $=0.2509 \mathrm{R}^{\mathrm{I}}$ \\
\hline $\mathbf{H}$ & 0.2372 & 0.2372 & \\
\hline \multirow[t]{2}{*}{$O$} & 45.8480 & 2.8720 & $=2.87200$ \\
\hline & 100.0000 & & \\
\hline
\end{tabular}

Wenn man nach dem Vorgange von RaMmeissberg den Borund Aluminiumgehalt als eine isomorphe Mischung betrachtet, so ergiebt sich aus den vorstehenden Analysen das folgende Verhältnis der den Axinit bildenden Elemente:

$$
\begin{aligned}
& \mathrm{Si}: \mathrm{R}^{\mathrm{III}} ; \mathrm{R}^{\mathrm{II}}: \mathrm{R}^{\mathrm{I}}: \mathrm{O} \\
& 2.83: 2.13: 2.08: 1: 11.44 \\
& 3: 2: 2: 1: 11^{1 / 2} \\
& 6: 4: 4: 2: 23
\end{aligned}
$$

welche Proportion der empirischen Formel:

entspricht.

$$
\mathrm{Si}_{6} \mathrm{O}_{23} \mathrm{R}_{4}^{\mathrm{II}} \mathrm{R}_{4}{ }_{4} \mathrm{R}_{2}
$$

Eine neuerdings in Aufnahme gekommene Formel des Axinits wurde von WHITFIELD, ${ }^{1}$ und zwar ebenfalls aus einer ausführlichen Analyse des Axinits aus der Dauphiné (Fundort Oisans) abgeleitet. Um seine Resultate bequemer mit den von uns erzielten vergleichen zu können, geben wir hier eine übersichtliche Zusammenstellung der von diesem Forscher mitgeteilten analytischen Werte:

1 Amer. Journ. of Seience [3] 34, 281. 


\begin{tabular}{|c|c|c|c|c|c|}
\hline & Analyse & $\begin{array}{c}\text { Auf } 100 \\
\text { berech- } \\
\text { net }\end{array}$ & & & Äquivalente \\
\hline $\mathrm{SiO}_{3}$ & 41.52 & 41.39 & $\mathrm{Si}$ & 19.3415 & $0.6908=0.6908 \mathrm{Si}$ \\
\hline $\mathrm{B}_{2} \mathrm{O}_{3}$ & 4.62 & 4.61 & B & 1.4420 & $0.1323=0.1323]^{B}$ \\
\hline $\mathrm{Al}_{2} \mathrm{O}_{3}$ & 17.90 & 17.84 & $\mathrm{Al}$ & 9.4623 & 0.3499 \\
\hline $\mathrm{Fe}_{2} \mathrm{O}_{3}$ & 3.90 & 3.89 & $\mathrm{Fe}^{\mathrm{III}}$ & 2.7297 & $0.3986] \mathrm{R}^{\mathrm{ML}}$ \\
\hline $\mathrm{FeO}$ & 4.02 & 4.01 & $F e^{1 I}$ & 3.1368 & 0.0559 \\
\hline $\mathrm{MnO}$ & 3.79 & 3.78 & $\mathrm{Mn}$ & 2.9272 & $0.5132 R^{\mathrm{II}}$ \\
\hline $\mathrm{CaO}$ & 21.66 & 21.59 & $\mathrm{Ca}$ & 15.4217 & 0.3864 \\
\hline $\mathrm{MgO}$ & 0.74 & 0.74 & $\mathrm{Mg}$ & 0.4440 & 0.0185 \\
\hline \multirow[t]{2}{*}{$\mathrm{H}_{2} \mathrm{O}$} & 2.16 & 2.15 & $\mathrm{H}$ & 0.2394 & $0.2394=0.2394 \mathrm{R}^{\mathrm{I}}$ \\
\hline & 100.31 & 100.00 & 0 & 44.8715 & $2.8115=2.81150$ \\
\hline
\end{tabular}

Zur Berechnung seiner Formel nahm WhitFIELd an, dafs das Bor und Aluminium nicht in gleicher Weise verbunden sind, und er fand, indem er das Boräquivalent als Einheit benutzte, die Verhältnisse :

$$
\mathrm{Si}: \mathrm{Al}: \mathrm{B}: \mathrm{Ca}^{\mathrm{FeMnMg}}: \mathrm{H}=5: 3: 1: 4: 2,
$$

aus welchen Zahlen er auf die Formel:

schlielst.

$$
\left(\mathrm{SiO}_{4}\right)_{5}(\mathrm{AlFe})_{3}(\mathrm{BO})(\mathrm{Ca} \cdots)_{4} \mathrm{H}_{2}
$$

Dieser Zusammensetzung nach stellt der Axinit ein Orthosilikat dar, welches die besondere einwertige Gruppe BO enthält. Wenn man aber unsere Resultate auf Grund derselben Annahme zu berechnen versucht, so ergiebt sich das folgende Sauerstoffverhältnis:

$$
\mathrm{Si}: \mathrm{Al}: \mathrm{B}: \mathrm{Ca} \cdots: \mathrm{H}=4.14: 2.1: 1: 3: 1.5,
$$

welches sich in keine Übereinstimmung mit der WHITFIELDschen bringen lärst. Die Möglichkeit seiner Formel hängt von einem konstanten Verhältnis zwischen Bor und den anderen dreiwertigen Elementen $a b . \quad O b$ eine derartige Gruppenbeziehung in Wirklichkeit besteht, ist an der Hand eines einzigen Mineralbeispieles gewils schwer zu entscheiden. Vergleicht man z. B. WhITFIELDs Resultate sowohl mit denjenigen von RAMMELSBERG, wie auch mit den unseren, so findet man das fragliche Verhältnis nicht konstant, sondern im Wechsel von $1: 3$ bis zu $1: 2$. Betrachtet man andererseits im Axinit alle Sesquioxyde als in isomorpher Mischung vorhanden, und berechnet daraufhin die Analyse Whitfields, so wird ein Verhältnis erhalten, welches sich sehr gut mit unseren Werten deckt: 


$$
\begin{aligned}
\mathrm{Si}: \mathrm{R}^{\mathrm{III}}: \mathrm{R}^{\mathrm{II}}: \mathrm{R}^{\mathrm{I}}: \mathrm{O} \\
2.89: 2.22: 2.14: 1: 11.72 \text { (W.) } \\
2.83: 2.13: 2.08: 1: 11.44 \text { (J. u. L.); }
\end{aligned}
$$

beide Analysen führen somit hier auf die gleiche Formel zurück.

Die von RAmmeLsBerg vorgeschlagene Formel:

$$
\left(\mathrm{SiO}_{4}\right)_{4} \mathrm{Al} \mathrm{H}_{2} \mathrm{~B}(\mathrm{Ca} \cdots)\left(\mathrm{Ca}^{\cdots}\right)_{3} \mathrm{H}^{1}
$$

\begin{tabular}{|c|c|c|c|c|c|c|}
\hline $\mathrm{SiO}_{2}$ & 43.46 & 42.99 & $\mathrm{Si}$ & 20.089 & \multicolumn{2}{|c|}{$0.7175=0.7175 \mathrm{Si}$} \\
\hline $\mathrm{B}_{2} \mathrm{O}_{3}$ & 5.61 & 5.55 & B & 1.736 & 0.1593 & \multirow[b]{2}{*}{$0.5108 \mathrm{R}^{\mathrm{yI}}$} \\
\hline $\mathrm{Al}_{2} \mathrm{O}_{8}$ & 16.33 & 16.15 & $\mathrm{Al}$ & 8.566 & 0.3168 & \\
\hline $\mathrm{Fe}_{2} \mathrm{O}_{3}$ & 2.80 & 2.77 & $\mathrm{Fe}^{\mathrm{III}}$ & 1.939 & 0.0347 & \multirow[t]{2}{*}{2} \\
\hline $\mathrm{FeO}$ & 6.78 & 6.71 & $\mathrm{Fe}^{\mathrm{II}}$ & 4.219 & 0.0755 & \\
\hline $\mathrm{MnO}$ & 2.62 & 2.59 & $\mathbf{M n}$ & 2.007 & 0.0364 & \multirow{2}{*}{$0.5318 \mathrm{R}^{\mathrm{If}}$} \\
\hline $\mathrm{CaO}$ & 20.19 & 19.99 & $\mathrm{Ca}$ & 14.279 & 0.3771 & \\
\hline $\mathrm{MgO}$ & 1.73 & 1.71 & $\mathrm{Mg}$ & 1.026 & 0.0428 & \multirow{3}{*}{$0.1614 \mathrm{R}^{\mathrm{I}}$} \\
\hline $\mathrm{K}_{2} \mathrm{O}$ & 0.11 & 0.11 & $\mathrm{~K}^{\circ}$ & 0.092 & 0.0024 & \\
\hline \multirow[t]{3}{*}{$\mathrm{H}_{2} \mathrm{O}$} & 1.45 & 1.43 & $\mathrm{H}$ & 0.159 & \multirow{3}{*}{\multicolumn{2}{|c|}{$2.8753=2.87530$}} \\
\hline & 101.08 & 100.00 & 0 & 45.888 & & \\
\hline & & & & 100.000 & & \\
\hline
\end{tabular}

wurde aus der folgenden Analyse abgeleitet. Wir geben auch dafür der besseren Übersicht wegen die zur Berechnung erforderlichen Daten:

Indem er die $\mathrm{Al}_{2}$-Äquivalente als Einheit benutzte, erhielt RAMMELsBerg die Quotienten:

$\mathrm{Si}: \mathrm{Al}: \mathrm{B}: \mathrm{Ca} \cdots: \mathrm{H}=4.1: 2: 1: 3: 1$

und damit die Formel:

$$
\left(\mathrm{SiO}_{4}\right)_{4} \mathrm{Al}_{2} \mathrm{~B}(\mathrm{Ca} \cdots)_{3} \mathrm{H} \text {. }
$$

Die gefundene Wassermenge ist nach der eigenten Ansicht des Forschers zu nieủrig. Um daher seine Resultate mit der obigen Formel in nächste Übereinstimmung zu bringen, nahm er an, dals die wirkliche Menge Wasser etwas grölser, nahe $2 \%$, wie er sagt, sei. Bei dieser Quantität Wasser entspricht seine Analyse nicht rollständig unserer Formel, besser jedoch für einen über $2 \%$ (etwa 2.15) hinausliegenden Wert. ${ }^{3}$

Die von uns vorgeschlagene Formel

$$
\mathrm{Si}_{6} \mathrm{O}_{28}(\mathrm{AlB})_{4}(\mathrm{CaFeMnMg})_{4} \mathrm{H}_{2}
$$

ist allgemein und damit vielleicht auch als annehmbar zu betrachten.

1 Mineralehemie 2. Auf. Seite 545.

${ }^{2} 0.1593(\mathrm{~B})+0.3515(\mathrm{Al}+\mathrm{Fe})$.

${ }^{3}$ Whitfieis fand $2.16 \%$ Wasser; die zur Bestimmung des Wassers benutzte Methode ist nicht angegeben. 
Der Axinit mufs nach derselben als eine intermediäre ${ }^{1}$ Verbindung, statt eines reinen Orthosilikates angesehen werden. Das Verhältnis $\mathrm{Si}: 0=6: 23$ ist dem des Nephelins ähnlich, in welchem Mineral $\mathrm{Si}: \mathrm{O}=9: 34$ figuriert. Die Zusammensetzung des Nephelins hat CLARKE $\cdot z u$ erklären versucht, indem er diese Verbindung als ein Orthosilikat darstellt, in welchem eine der Aluminium-Valenzen durch die einwertige Gruppe $\mathrm{SiO}_{3} \mathrm{Na}$ gesättigt ist. Denkt man sich in dem Axinit dieselbe Gruppe eingelagert, so kann man die Formel schreiben:

oder graphisch

$$
\left(\mathrm{SiO}_{4}\right)_{5}(\mathrm{AlB})_{3}\left(\mathrm{AlSiO}_{3} \mathrm{H}\right)(\mathrm{Ca} \cdots)_{4} \mathrm{H}
$$

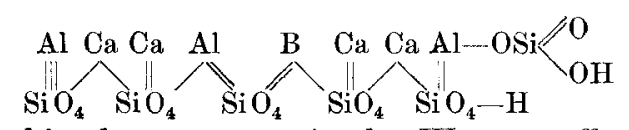

In der Metakieselsäuregruppe ist der Wasserstoff teilweise durch die Alkalimetalle vertreten; in der Orthokieselsäure sind Aluminium und Bor isomorph und das zweiwertige Calcium wird zum Teil durch Eisen, Mangan und Magnesium ersetzt. Wenn $\mathrm{Al}: \mathrm{B}=2: 1$ ist, wie solches in dem von uns analysierten Vorkommnis der Fall war, alsdann entsprechen unserer Formel die folgenden, durchschnittlich gut mit den gefundenen Resultaten übereinstimmenden Werte:

$$
\begin{aligned}
& \mathrm{SiO}_{2}=45.80 \%{ }^{1} \\
& \mathrm{Al}_{2} \mathrm{O}_{3}=17.40 \% \\
& \mathrm{~B}_{2} \mathrm{O}_{3}=5.90 \% \\
& \mathrm{CaO} \\
& \left.\begin{array}{l}
\mathrm{FeO} \\
\mathrm{MnO}
\end{array}\right\}=28.60 \% \\
& \mathrm{MgO}) \\
& \mathrm{H}_{2} \mathrm{O}=2.30 \%
\end{aligned}
$$

Wir haben auch versucht, die Formel ohne Berücksichtigung der Alkalien zu berechnen, ferner in der Voraussetzung, dafs dieselben event. als Vertreter des Calciums vorhanden seien; allein in beiden Fällen gelangten wir auf keine annehmbare Formel. ${ }^{3}$

${ }^{1}$ Groth, Tabellarische Übersicht der Mineralien, Seite 123.

2 Da man bei dieser Sachlage die zweiwertigen Elemente alle als $\mathrm{Ca}$, ferner die dreiwertigen als Aluminium berechnen muls, was auf die ganze Gruppe bezogen ein zu niedriges Atomgewicht ergiebt, so stellen sich natürlich die so erhaltenen Kieselsäureprozente erheblich höher, als den faktischen Verhältnissen in Wirklichkeit entspricht.

${ }^{3}$ Ganz neuerdings hat H. RHEINEck in GRoths Zeitsehr. Krystallogr. 22, 275, den Versuch gemacht, sämtliche bislang aufgestellte Axinit-Formeln 
Eine von uns geplante Ausdehnung unserer chemischen Untersuchung auf eine Reihe anderer Axinitvorkommnisse wird sicherlich weitere feste Stützpunkte und Einblicke bringen zur Erlangung eines allgemeineren Urteiles über die Konstitutionsverhältnisse des noch zu selten analysierten Boro-Silikates.

von Rammelsberct, Whitfiecd, Genth und Baumert auf Mischungsverhältnisse zurückzuführen, welche sich durch folgende zwei Schemata ausdrücken lassen:

$$
\begin{aligned}
& \mathrm{Al}_{2} \mathrm{O}_{4}=\mathrm{Si}_{4} \mathrm{O}_{5}=\mathrm{O}_{5} \mathrm{Ca}_{2} \mathrm{BH}=\mathrm{O}_{2} \mathrm{Fe} \text { und } \\
& \text { VIII } \\
& \mathrm{Al}_{2} \mathrm{O}_{4}=\mathrm{Si}_{4} \mathrm{O}_{4}=\mathrm{O}_{5} \mathrm{Ca}_{2} \mathrm{BH}=\mathrm{O}_{2} \mathrm{Fe}=\mathrm{O}_{2} \mathrm{Ca} .
\end{aligned}
$$

Heidelberg, Universitätslaborat., Februar 1894.

Eingegangen bei der Redaktion am 2. Februar 1894. 